\title{
Native nephrectomy in transplant patients with autosomal dominant polycystic kidney disease
}

\author{
P Patel, C Horsfield, F Compton, J Taylor, G Koffman, J Olsburgh
}

\author{
Guy's Hospital, London, UK
}

ABSTRACT

INTRODUCTION This study examined the clinical indications and timing for native nephrectomy (NN), together with the associated pathological findings in transplant patients with autosomal dominant polycystic kidney disease (ADPKD) at our institute over a period of 20 years.

METHODS A retrospective review was performed of ADPKD patients who had undergone both kidney transplantation and NN. Patients were identified from the kidney transplant database between 1988 and 2008 at Guy's and St Thomas' Hospital and the notes reviewed. All NN specimens were re-reviewed and reported according to current guidelines.

RESULTS There were 157 kidney transplants performed for ADPKD (114 cadaveric and 43 living donor). Of these, 31 required NN (28 bilateral). The timing of NN was pre-transplant in 10 cases, at the time of the transplant in 1 case and posttransplant in 20 cases. The indications for NN were urinary tract infection $(n=14,45 \%)$, pain $(n=12,39 \%)$, tumour suspicion $(n=3,10 \%)$, haematuria $(n=1,3 \%)$ and space $(n=1,3 \%)$. Mortality in this NN series was $3 \%$, with a $65 \%$ surgical morbidity rate. The length of hospital stay post-NN was significantly longer with open compared with laparoscopic techniques $(p=0.003)$. There were two renal cell carcinomas (RCCs) in this series. Both patients presented with macroscopic haematuria (bilateral pT1a papillary RCCs in one case and a pT3b clear cell RCC in the other case). The incidence of RCC in this series of ADPKD transplant patients was $1.3 \%$.

CONCLUSIONS We have demonstrated that the majority of ADPKD patients do not require NN, with only $20 \%$ of our series undergoing this procedure. The timing of NN is variable and dictated by indication. NN was only required to make space for transplantation in one case (combined kidney and pancreas transplant). The main indications for NN were recurrent infection and pain, where NN can provide a successful outcome. Laparoscopic NN can be performed safely in patients with ADPKD. Haematuria in such patients should not be assumed to be of benign origin and requires exclusion of urinary tract malignancy as the incidence of RCC in this population is at least as common as in the general population.

KEYWORDS

Polycystic - Kidney - Transplant - Native - Nephrectomy

Accepted 24 April 2011

CORRESPONDENCE TO

Jonathon Olsburgh, Consultant Transplant and Urological Surgeon, Directorate of Urology, Nephrology and Transplantation, Guy's Hospital, Great Maze Pond, London SE1 9RT, UK

E: jonathon.olsburgh@gstt.nhs.uk

Autosomal dominant polycystic kidney disease (ADPKD) is a dominantly inherited heterogenetic systemic disease occurring in 1:700 to 1:1,000 individuals. It is one of the most common inherited disorders with approximately 12.5 million cases worldwide. ${ }^{1}$ The development of multiple epitheliallined kidney cysts results in slowly progressive enlargement of the kidney that leads to chronic kidney disease (CKD) in $50 \%$ of patients by the sixth decade. ${ }^{1,2}$ Cysts can also develop in other organs including the liver $(80 \%)$, pancreas $(10 \%)$ and, more rarely, the spleen and seminal vesicles. ${ }^{3}$ ADPKD is responsible for approximately $6-10 \%$ of CKD cases in North America and Europe. ${ }^{4-6}$ Patients may present with haematuria, a urinary tract infection (UTI), loin pain, an abdominal mass, CKD or hypertension.

Kidney transplantation is the preferred treatment option in ADPKD patients with CKD. Patients with recurrent infec- tion, intractable loin pain, persistent haematuria and suspected malignancy may require native nephrectomy $(\mathrm{NN})$. There is little consensus on the timing or indications for NN in ADPKD kidney transplant recipients. We have evaluated our single centre series over the last 20 years.

\section{Methods}

A retrospective review was performed of the kidney transplant and histopathology databases at Guy's and St Thomas' hospitals covering 1988-2008. ADPKD patients who had undergone both kidney transplantation and NN were selected for our case review. Histopathology reports of NN specimens were re-reviewed according to current guidelines by a single consultant renal pathologist. Specific data relating to indication, timing and pathology of $\mathrm{NN}$ were collected 


\begin{tabular}{|c|c|c|}
\hline Pathological group & Timing of NN & Indication for NN \\
\hline $\begin{array}{l}\text { Renal cell } \\
\text { carcinoma }(n=2)\end{array}$ & $\begin{array}{l}\text { Post-transplant } \\
(n=2) \\
4 \text { and } 6 \text { years }\end{array}$ & $\begin{array}{l}\text { Tumour suspicion } \\
(n=2)\end{array}$ \\
\hline \multirow{2}{*}{$\begin{array}{l}\text { Benign tumour } \\
(n=5)\end{array}$} & Pre-transplant $(n=2)$ & UTI $(n=2)$ \\
\hline & $\begin{array}{l}\text { Post-transplant } \\
(n=3) \\
1,8 \text { and } 17 \text { years }\end{array}$ & $\begin{array}{l}\text { UTI }(n=1) \\
\text { Pain }(n=2)\end{array}$ \\
\hline \multirow[t]{3}{*}{$\begin{array}{l}\text { Autosomal dominant } \\
\text { polycystic kidney } \\
\text { disease }(n=24)\end{array}$} & Pre-transplant $(n=8)$ & $\begin{array}{l}\text { UTI }(n=2) \\
\text { Pain }(n=5) \\
\text { Haematuria }(n=1)\end{array}$ \\
\hline & $\begin{array}{l}\text { Post-transplant } \\
(n=15) \\
0.5-12.5 \text { years }\end{array}$ & $\begin{array}{l}\text { UTI }(n=9) \\
\text { Pain }(n=5) \\
\text { Tumour suspicion } \\
(n=1)\end{array}$ \\
\hline & Simultaneous $(n=1)$ & Space $(n=1)$ \\
\hline
\end{tabular}

from patient medical records. Indications for NN were categorised into pain, UTI, haematuria (macroscopic), tumour suspicion (computed tomography findings suggestive of malignancy) and space (for transplantation). Surgical morbidity was categorised using the Clavien system. ${ }^{7}$ Statistical analyses were performed using chi-squared, Wilcoxon signed-rank and Mann-Whitney U tests to compare categorical variables, with a $p$-value of $<0.05$ considered significant.

\section{Results}

Between 1988 and 2008, 1,712 adult patients underwent kidney transplantation at our institute and ADPKD was attributed as the cause of CKD in 157 (8\%). Of these patients, 79 were female and 78 male; $114(73 \%)$ received a cadaveric kidney transplant and 43 a living donor kidney transplant. NN was performed in 31 patients $(20 \%)$, (12 female and 19 male, mean age: 49 years). The majority $(n=28)$ had bilateral NN; 10 were performed pre-transplant and 18 posttransplant. The indications for bilateral NN were: haematuria $(n=1)$, tumour suspicion $(n=3)$, recurrent UTI $(n=12)$ and pain $(n=12)$. Three patients underwent unilateral NN. Two of these were performed post-transplant for a recurrent UTI and one (right NN) was performed for space simultaneously with a combined kidney and pancreas transplant.

Fourteen patients (seven male) underwent NN for recurrent UTIs: four pre-transplant, ten post-transplant. Eleven of these patients had resolution of their recurrent UTI immediately after surgery. The other three patients (one male) had documented symptoms and/or positive mid-stream urine cultures after NN, with one patient experiencing a recurrent $(\geq 3)$ UTI. In these three cases NN was performed pre-transplant in one patient ( 0.2 years) and post-transplant in two patients (12.5 years and 5.5 years).

There were two cases of renal cell carcinoma (RCC): bilateral pT1a Nx Mx papillary RCCs in one case and a pT3b Nx Mx (Fuhrman grade 2) clear cell RCC in the other. There

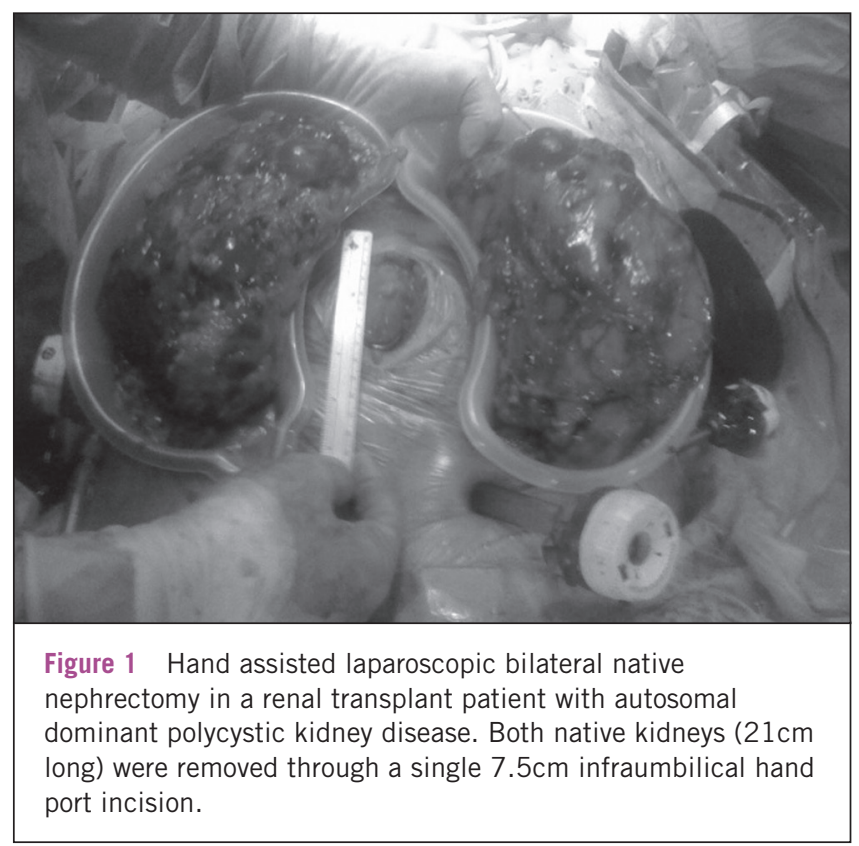

were five cases with benign renal tumours, five papillary adenomas (two found in one specimen) and one oncocytoma. In contrast, 24 of the 31 cases that underwent NN only showed histological features consistent with ADPKD. The incidence of RCC in this ADPKD transplant series was 1.3\%.

The correlation between the indication for $\mathrm{NN}$ and the pathological findings is shown in Table 1. Both cases of RCC presented with macroscopic haematuria and had imaging suggestive of a tumour. Two other cases presented with haematuria but only had pathological features of uncomplicated ADPKD. The imaging in one of these cases was suggestive of a tumour. The benign tumours were incidental pathological findings, with NN being performed for either pain or a UTI. Pain and UTIs were the main indications for $\mathrm{NN}$, accounting for $84 \%$ of cases.

Table 1 also summarises the timing of $\mathrm{NN}$ in this series. For the two cases with malignant pathology, NN was performed at four and six years post-transplant. For the benign pathology cases, NN was performed pre-transplant in 10 cases, simultaneously with the transplant in 1 case and posttransplant in 18 cases.

The exposure time to dialysis of the native kidneys with malignant pathological findings was 11 and 12 months. For those with benign tumours, the median exposure time was 12 months (range: 0.5-36 months) and for those with ADPKD findings alone, it was also 12 months (range: 0-78 months).

The surgical technique used for NN in this series was open (midline or rooftop) in the majority $(n=26)$ of cases. In the five most recent cases the kidneys were removed laparoscopically with three hand assisted laparoscopic (HAL) bilateral NNs, one HAL unilateral NN and one total laparoscopic unilateral NN. The paired kidneys in the HAL NN cases were between $20 \mathrm{~cm}$ and $30 \mathrm{~cm}$ in length and were extracted via a subumbilical hand port site (Fig 1).

The mortality rate for this series was $3 \%(1 / 31$, Clavien grade 5), with an overall surgical complication rate of $65 \%$ (20/31, Clavien grades 1-5). The incidence of surgical 
Hajj et al, 200912 79 ADPKD patients with CKD had NN between 1982 and 2003 (10 bilateral)

Indication: tumour suspicion ( $n=5)$, space or benign complications (haematuria, pain, infection)

Histology: RCC ( $n=10,5$ clear cell carcinomas and 7 tubulopapillary carcinomas)

The prevalence of RCC was found to be higher in patients with ADPKD and CKD, with >1 year on dialysis or kidney transplantation.

Nunes et al, 200913 159 kidney transplant patients with ADPKD

143 patients (90\%) had simultaneous unilateral NN at the time of transplant for space

Cohen et al, $2008^{9}$

145 patients with ADPKD between 1998 and 2006

NN performed in 38 patients

Indication: in preparation for transplant $(n=28,68 \%)$, urological complications $(n=12)$, tumour suspicion $(n=1)$

Lipke et al, $2007^{14}$

Outcome review of 18 patients with ADPKD who underwent hand assisted laparoscopic bilateral NN

Timing of NN: post-transplant $(n=17)$

Indication: pain ( $n=16)$, recurrent infection $(n=2)$

Fuller et al, $2005^{8}$

171 ADPKD patients with CKD between 1992 and 2002

NN performed in 32 transplant patients (25 bilateral)

Timing of NN: pre-transplant $(n=7)$, simultaneous ( $n=16)$, post-transplant $(n=9)$

Indication: pre-transplant for haematuria, pain or mass; simultaneously for space; post-transplant for UTI

Hadimeri et al, 1997 11 874 kidney transplant patients between 1985 and 1993

114 (13\%) for ADPKD

NN performed in 26 patients

Timing of NN: pre-transplant ( $n=19)$, post-transplant $(n=7)$

Indication: pre-transplant for space $(n=11)$, infection/pain/haematuria $(n=5)$, tumour suspicion $(n=2)$, hypertension $(n=1)$; post-transplant for space $(n=1)$

Keith et al, $1994^{15}$

25 patients with ADPKD and RCC at Mayo Clinic between 1955 and 1992

3 cases bilateral RCC, 2 cases developed RCC in contralateral kidney, 7 cases of multicentric tumours and $20 \%$ had metastases at presentation

There was a higher proportion of sarcomatoid tumour compared to the general population.

Our institute, 2010

157 ADPKD patients between 1988 and 2008 underwent kidney transplantation

NN performed in 31 patients (20\%) (28 bilateral)

Timing of NN: pre-transplant $(n=10)$, simultaneous $(n=1)$, post-transplant $(n=20)$

Indication: haematuria $(n=1)$, tumour suspicion $(n=3)$, UTI $(n=14)$, pain $(n=12)$, space $(n=1)$

Histology: RCC in 2 cases (incidence 1.3\%): 1 bilateral papillary RCC and 1 clear cell RCC

$\mathrm{CKD}=$ chronic kidney disease; $\mathrm{RCC}=$ renal cell carcinoma; UTI = urinary tract infection

complications in patients who underwent open NN was $73 \%$ (median: grade 2, range: grades 1-5) and for laparoscopic NN it was $20 \%$ (a single grade 4 complication). The average number of complications per patient in the open NN group was 1.1 compared with 0.2 in the laparoscopic NN group.

There was no significant difference in the complication rate between NN performed pre-transplant (65\%) and those performed post-transplant $(61 \%)(p=0.55)$. However, there was a significant difference in the severity of complications between these two groups, with all major complications (grade 3-5) occurring in those NNs performed post-transplant $(p=0.034)$.

The majority of the NNs were performed in an elective 
setting. There were five emergency open NN cases: one pre-transplant, one simultaneous and three post-transplant. The indications were infection $(n=4)$ and space at the time of a combined kidney and pancreas transplant $(n=1)$. All had postoperative complications. One emergency laparoscopic NN was performed for a UTI; no postoperative complications were noted.

The median length of hospital stay (LOS) after NN in this series was 12 days (range: 5-58 days). For the open NN group the median LOS was 12 days (range: 7-59 days) and for the laparoscopic (HAL/total laparoscopic) NN group the median LOS was 5 days (range: $5-12$ days) $(p=0.003)$. For those who underwent emergency open NN, the median LOS was 21.5 days (range: $12-58$ days) whereas the LOS for the one emergency laparoscopic NN was 5 days.

\section{Discussion}

There is no clear consensus as to the exact indications or timing for NN in the ADPKD population undergoing transplantation. Most would agree that NN is not routinely indicated in this population, with the majority of patients not requiring $\mathrm{NN}$ as demonstrated in our series where $20 \%$ of our ADPKD population underwent $\mathrm{NN}$ and also those studied by Fuller et al $(\mathrm{NN}=19 \%),{ }^{8}$ Cohen et al $(\mathrm{NN}=26 \%),{ }^{9} \mathrm{Su}-$ likowski et al $(\mathrm{NN}=16 \%)^{10}$ and Hadimeri et al $(\mathrm{NN}=17 \%)^{11}$ who performed similar numbers (Table 2 ).

Whereas in those series by Fuller et al, Cohen et al and Hadimeri et al the main indication for $\mathrm{NN}$ was to provide space for the transplant, we have shown this to be unnecessary in most cases. Only one patient in our series underwent NN for space; this was for a combined kidney and pancreas transplant where the right kidney was removed to provide space for the pancreas transplant.

The main indications for $\mathrm{NN}$ in our series were recurrent infection or intractable pain (84\% of cases) and NN was mostly performed post-transplant. Patients are at highest risk of a UTI in the first month post-transplant and it is the most common infection to be reported following kidney transplantation. The reported incidence of post-transplant UTIs varies considerably from $10 \%$ to $98 \%{ }^{16}$ Many factors have been attributed to the increased incidence of UTIs in kidney transplant patients. Transplant ureteric stents and instrumentation of the urinary tract predispose to infection. In this patient series the majority had a transplant ureteric stent for 6-12 weeks before being removed cystoscopically. Immunosuppression is an important risk factor for infection in transplant patients and is at highest levels during the first year post-transplant.

In the three patients with persistent UTIs post-NN, all the infections were noted to occur post-transplant. One was managed by a reduction in immunosuppressant dose, suggesting excessive immunosuppression was a contributing factor. Urinary stasis is also a recognised risk factor for UTIs and incomplete bladder emptying was found to be the cause in one patient with a persisting UTI post-NN. The UTI in this patient resolved after adopting a double voiding regime. Women are predisposed to an increased risk of UTI as seen in our cohort of patients where the main indication for $\mathrm{NN}$ in our female population was UTI $(58 \%)$.

NN performed for UTIs resolved the problem immediately in $75 \%$ of our cases and by 1 year post-NN all 14 patients were UTI free. It is important to remember that the cause of UTIs in ADPKD patients is not always in the native kidneys and NN cannot resolve lower urinary tract problems in these patients. This highlights the importance of preoperative investigations and careful patient selection prior to NN. Investigations prior to NN for UTIs should include imaging of the urinary tract, ultrasound measurement of pre- and post-urination bladder volumes, urinary flow rate and and cystoscopy. Occasionally, positron emission tomography - computed tomography is indicated to localise infection to either liver or renal cysts in ADPKD. ${ }^{17}$

Approximately two-thirds of patients with ADPKD develop micro- or macroscopic haematuria, which is most often due to UTIs, cyst rupture or stone disease. ${ }^{18,19}$ There has been ongoing controversy as to whether ADPKD is a risk factor for RCC. While the literature is inconclusive with regards to this, it seems that malignancy is at least as common in this subgroup as in the general population..$^{12,15,20}$ In our study, the incidence of RCC in NN specimens was $1.3 \%$, which is equivalent to that in the general population. ${ }^{21-24}$ The two patients with RCC were aged 53 and 62 years and both presented with macroscopic haematuria. This study confirms that haematuria cannot be presumed to be due to a benign cause in ADPKD patients and that urinary tract malignancy must be excluded.

In addition to the three RCCs (bilateral in one case), histological examination of our NN specimens revealed five papillary adenomas. The concept of a papillary adenoma is controversial and in theory related to a proliferation in tubulopapillary epithelium that has no metastatic potential. Nevertheless, they are very similar histomorphologically to papillary RCCs..$^{25}$ It has been suggested that as the size of papillary adenomas increases so does the amount of chromosomal alteration and the potential to transform to cancer. This raises the possibility that papillary adenomas and papillary RCCs represent a continuum of the same process. The World Health Organization has defined size criteria to differentiate them, with papillary adenomas being $<5 \mathrm{~mm}$ and carcinomas $\geq 5 \mathrm{~mm} .{ }^{26}$

Acquired cystic kidney disease (ACKD) increases with time on dialysis, affecting approximately $50 \%$ of those on dialysis at three years. ACKD is associated with an increased incidence (4-20\%) of RCC. ${ }^{27-30}$ In our study, we did not find that the malignant pathological group had spent a longer period on dialysis compared with the benign group. This might suggest that the RCCs did not develop as a result of time spent on dialysis. It is difficult to determine whether ACKD co-exists in ADPKD kidneys with standard histopathology. We were therefore not able to confirm if there were ACKD findings in either the benign or malignant ADPKD groups.

Most studies have shown that unilateral or bilateral NN in ADPKD patients is safe in terms of postoperative morbidity and graft function ${ }^{8-10,15}$ and our morbidity and mortality rates compared to those in other series. The one mortality in this series was a patient who underwent $\mathrm{NN}$ as part of an 
emergency laparotomy for overwhelming sepsis. The Clavien classification is a validated system for stratifying surgical complications and enabled complications to be categorised into 'minor' (grades 1 and 2) and 'major' (grades 3-5). ${ }^{7}$ As expected, $\mathrm{NN}$ in the emergency setting was associated with more severe complications. Interestingly, we found that all of the major complications occurred in those NNs performed post-transplant and may relate to the immunesuppressed state. Avoiding the need for emergency NN, particularly in the post-transplant period when complications appear to be more severe, may reduce the postoperative morbidity, LOS and mortality.

The majority of our nephrectomies were performed with a midline or rooftop open incision. The five most recent cases utilised laparoscopic techniques and, similar to the authors of other studies, we believe that laparoscopic NN techniques show promising benefits in terms of decreased morbidity, blood loss and hospital stay despite being a technically challenging procedure.$^{14,31-35}$ Further studies are needed to confirm the potential benefits of laparoscopy compared with open nephrectomy in this patient group.

The major limitation of this study is that we did not review the records of the 128 ADPKD transplant patients who did not require $\mathrm{NN}$ and are therefore unable to comment on the overall incidence of haematuria, infection and pain in these patients.

\section{Conclusions}

Haematuria must be investigated fully and promptly in the ADPKD population, as one would for the general population, to exclude a renal tract malignancy. $\mathrm{NN}$ is not routinely indicated in ADPKD patients undergoing transplantation but is reserved mainly for those with recurrent infection and pain. The majority of NN performed for UTIs successfully prevented further episodes of infection, demonstrating that in a select group NN can improve morbidity. Studies suggest that the timing of NN does not significantly impact on morbidity or graft survival. Minimally invasive NN surgery appears to be a useful development in ADPKD patients.

\section{Acknowledgements}

To the ADPKD transplant patients at Guy's and St Thomas' hospitals and the medical and nursing staff involved in their care.

\section{References}

1. US Renal Data System 2008 Annual Data Report: Atlas of Chronic Kidney Disease and End-Stage Renal Disease in the United States. Bethesda, MD: USRDS; 2008.

2. Chapman AB. Approaches to testing new treatments in autosomal dominant polycystic kidney disease: insights from the CRISP and HALT-PKD studies. Clin J Am Soc Nephrol 2008; 3: 1,197-1,204.

3. Chapman AB. Autosomal dominant polycystic kidney disease: time for a change? J Am Soc Nephrol 2007; 18: 1,399-1,407.

4. Zeier M, Jones E, Ritz E. Autosomal dominant polycystic kidney disease - the patient on renal replacement therapy. Nephrol Dial Transplant 1996; 11: 18-20.

5. Wing AJ, Brunner FP, Brynger $\mathrm{H}$ et al. Combined report on regular dialysis and transplantation in Europe, VIII, 1977. Proc Eur Dial Transpl Assoc 1978; 15: $2-76$.

6. Simon P, Le Goff JY, Ang KS et al. Epidemiologic data, clinical and prognostic features of autosomal dominant polycystic kidney disease in a French region. Nephrologie 1996; 17: 123-130.

7. Dindo D, Demartines N, Clavien PA. Classification of surgical complications: a new proposal with evaluation in a cohort of 6336 patients and results of a survey. Ann Surg 2004; 240: 205-213.

8. Fuller TF, Brennan TV, Feng $S$ et al. End stage polycystic kidney disease: indications and timing of native nephrectomy relative to kidney transplantation. J Urol 2005; 174: 2,284-2,288.

9. Cohen D, Timsit MO, Chrétien $Y$ et al. Place of nephrectomy in patients with autosomal dominant polycystic kidney disease waiting for renal transplantation. Prog Urol 2008; 18: 642-649.

10. Sulikowski T, Teijchman K, Zietek Z et al. Experience with autosomal dominant polycystic kidney disease in patients before and after renal transplantation: a 7-year observation. Transplant Proc 2009; 41: 177-180.

11. Hadimeri H, Nordén G, Friman S, Nyberg G. Autosomal dominant polycystic kidney disease in a kidney transplant population. Nephrol Dial Transp/ 1997; 12: 1,431-1,436.

12. Hajj P, Ferlicot S, Massoud W et al. Prevalence of renal cell carcinoma in patients with autosomal dominant polycystic kidney disease and chronic renal failure. Urology 2009; 74: 631-634.

13. Nunes, Moto $A$, Alves $R$ et al. Simultaneous renal transplantation and native nephrectomy in patients with autosomal-dominant polycystic kidney disease. Transplant Proc 2007; 39: 2,483-2,485.

14. Lipke MC, Bargman V, Milgrom M, Sundaram CP. Limitations of laparoscopy for bilateral nephrectomy for autosomal dominant polycystic kidney disease. J Urol 2007; 177: 627-631.

15. Keith DS, Torres VE, King BF et al. Renal cell carcinoma in autosomal dominant polycystic kidney disease. J Am Soc Nephrol 1994; 4: 1,661-1,669.

16. de Souza RM, Olsburgh J. Urinary tract infection in the renal transplant patient. Nat Clin Pract Nephrol 2008; 4: 252-264.

17. Desouza RM, Prachalias A, Srinivasan P et al. Differentiation between infection in kidney and liver cysts in autosomal dominant polycystic kidney disease: use of PET-CT in diagnosis and to guide management. Transplant Proc 2009; 41 : $1,942-1,945$

18. Dedi R, Bhandari S, Turney JH et al. Causes of haematuria in adult polycystic kidney disease. BMJ 2001; 323: 386-387.

19. Gabow PA, Duley I, Johnson AM. Clinical profiles of gross hematuria in autosomal dominant polycystic kidney disease. Am J Kidney Dis 1992; 20 140-143.

20. Nishimura $\mathrm{H}$, Ubara $\mathrm{Y}$, Nakamura $\mathrm{M}$ et al. Renal cell carcinoma in autosomal dominant polycystic kidney disease. Am J Kidney Dis 2009; 54: 165-168.

21. Kidney cancer - UK mortality statistics. Cancer Research UK. http://info. cancerresearchuk.org/cancerstats/types/kidney/mortality/ (cited May 2011).

22. Black RJ, Bray F, Ferlay J, Parkin DM. Cancer incidence and mortality in the European Union: cancer registry data and estimates of national incidence for 1990. Eur J Cancer 1997; 33: 1,075-1,107.

23. Mindrup SR, Pierre JS, Dahmoush L, Konety BR. The prevalence of renal cell carcinoma diagnosed at autopsy. BJU Int 2005; 95: 31-33.

24. McLaughlin JK, Lipworth L, Tarone RE. Epidemiologic aspects of renal cell carcinoma. Semin Oncol 2006; 33: 527-533.

25. Wang KL, Weinrach DM, Luan C et al. Renal papillary adenoma -a putative precursor of papillary renal cell carcinoma. Hum Pathol 2007; 38: 239-246.

26. Algaba F. Renal adenomas: pathological differential diagnosis with malignant tumors. Adv Urol 2008; 974848.

27. Matson MA, Cohen EP. Acquired cystic kidney disease: occurrence, prevalence, and renal cancers. Medicine (Baltimore) 1990; 69: 217-226.

28. Stewart JH, Buccianti G, Agodoa $L$ et al. Cancers of the kidney and urinary tract in patients on dialysis for end-stage renal disease: analysis of data from the United States, Europe, and Australia and New Zealand. J Am Soc Nephrol 2003; 14: 197-207.

29. Schwarz A, Vatandaslar S, Merkel S, Haller H. Renal cell carcinoma in transplant recipients with acquired cystic kidney disease. Clin J Am Soc Nephrol 2007; 2: 750-756.

30. Noronha IL, Ritz E, Waldherr $\mathrm{R}$ et al. Renal cell carcinoma in dialysis patients with acquired renal cysts. Nephrol Dial Transplant 1989; 4: 763-769.

31. Rehman J, Landman J, Andreoni C et al. Laparoscopic bilateral hand assisted nephrectomy for autosomal dominant polycystic kidney disease: initial experience. J Urol 2001; 166: 42-47.

32. Dunn MD, Portis AJ, Elbahnasy AM et al. Laparoscopic nephrectomy in patients with end-stage renal disease and autosomal dominant polycystic kidney disease. Am J Kidney Dis 2000; 35: 720-725.

33. Desai MR, Nandkishore SK, Ganpule A, Thimmegowda M. Pretransplant laparoscopic nephrectomy in adult polycystic kidney disease: a single centre experience. BJU Int 2008; 101: 94-97.

Ann R Coll Surg Engl 2011; 93: 391-395 